\title{
Benchmarking of AODV Routing Protocol Implemented for Military Software Defined Radio Waveform
}

\author{
Harvaneet Kaur \\ Student \\ Dept. Of EECE, ITM University \\ Gurgaon, Haryana
}

\author{
Amanpreet Kaur \\ Assistant Professor \\ Dept. Of EECE, ITM University \\ Gurgaon, Haryana
}

\author{
Dhiraj Khanna \\ Senior System Manager \\ Ministry Of Defence \\ New Delhi
}

\begin{abstract}
Military wireless networks used for tactical communication are classified as MANETS. These are dynamic, infrastructureless networks that are well suited for Line of Sight (LOS) as well as Beyond-Line of sight (BLOS) communication. This research work benchmarks the performance of the routing protocol implemented for a high data rate (up to $6 \mathrm{Mbps}$ ) $\mathrm{L}$ band waveform against the famous AODV routing protocol for wireless networks. Similarity in design of the SDR routing protocol to AODV is the reason for benchmarking against AODV. This paper will first explain about the software defined radios (SDR) followed by the different routing protocols for MANET. Based on the selection of a particular routing protocol different performance parameters that are Packet delivery ratio, Packet Loss, Routing overhead and Throughput have been calculated. Finally, based on the simulated results a conclusion has been drawn to improve the performance of SDR.
\end{abstract}

\section{Keywords}

MANETS, SDR, AODV.

\section{INTRODUCTION}

In a tech-savvy world personal computer has become an integrated part of the modern life and this led to the foundation of the software defined radio (SDR) [1]. The hardware required for personal computer has been a common platform, wherein the software has been designed to compensate with the hardware differences. Still Hardware is considered more common, whereas the software has become more scalable as well as more flexible. Any kind of change in the technology can be straight away made to the software which can later be loaded into the radio system. Software define radio (SDR) is an emerging technology in military communication. This new approach supports multiple channels with portable waveforms that provide higher level flexibility and scalability to the user, thereby providing a quick technology insertion whenever there is the need.

SDR uses digital data which it obtains from the analog signals and then it processes it by different resources that are there within the radio. The software maintains different aspects of the Radio frequency and provides a man-machine interface along with this. Some of the advantages of SDR over legacy radios are:

- Flexibility in designing various communication components by using the software.

- Since a SDR has more software components vis-àvis conventional radios, the MTBF (mean time between failures) is considerably higher than conventional radios. Thus SDRs have far greater reliability as compared to legacy radios.

- Consistency and stability of the parameters are more efficient in SDR and thus improves its performance and thereby reduces the aging effects as well.

- Upgradability, reusability, configurability and enhanced functionality are some of the other considerable advantages of SDR.

- $\quad$ Since multiple waveforms can be loaded on a single SDR, it gives user the advantage of having multiple radios at the cost of one. Hence SDR is a very cost effective solution in a military scenario, which requires the use of different waveform/radios for different scenarios.

SDR technology has made inroads not only in military tactical communication, but also for civil application. This research work is related to the indigenous SDR programme being implemented jointly by Indian Navy and DRDO. While most of the information under this is confidential, some of the salient features are:

- Standard software architecture which is based on SCA.

- Reconfigurable, scalable hardware platform, with the operational frequency range from $3 \mathrm{MHz}-$ $1.24 \mathrm{GHz}$ for tactical usage.

- Portable waveforms.

- Software defined and hardware secured cryptography.

- Integrated communication node based on the internet protocol.

- Broad spectrum coverage.

- Interference mitigation.

- Use of open system architecture.

The application of SDR in military is highly motivated by the network-centric operations which are advantageous in following perspective:

- $\quad$ SDR is the key element for securely and robustly networking the forces.

- The SDR provides both new, IP-capable waveforms as well as interoperability with legacy waveforms.

- SDR links the highly mobile units to the communication networks. 


\section{WIRELESS NETWORKING PROTOCOLS}

MANET [2] which is the prominent technology allowing its users to communicate independent of any infrastructure and also of their geographical positions. Due to this sometimes these are also considered as infrastructure-less networks. These are self-adaptive self-healing and self-organizing and perform sufficient operations for carrying out communication between the sources and the destinations. Ad-Hoc network allows an easy access for the other devices to either become a part of the network or to leave the network without affecting the overall performance of the network.

There are many metrics that determine the performance of the ad-hoc networks and these are:

- Signal to noise ratio

- Transmission queue size

- Location update

- Speed of operation

- Routing

- Network congestion

- Packet loss

The performance of a wireless data link is actually affected by many factors [3] like node mobility, fading bandwidth etc. Apart from having to deal with these factors, military wireless networks are extremely sensitive to bandwidth usage. Bandwidth not only needs to be optimally utilized, but usage needs to be minimum to minimize adversary effects such as jamming. Hence, unlike a conventional network, in a military network, exchange of control packets for maintaining a network needs to be highly optimized along with built in robustness against jamming achieved through LPI (low probability of intercept) features.

Routing protocols in MANETs are broadly classified as:

- PROACTIVE PROTOCOL

Commonly referred as the table driven protocol, is the routing protocol wherein the routing tables for all the routes are maintained previously thereby consuming a lot of bandwidth. Since the routing tables are already maintained, therefore, the latency of this is also less and also the information is always available. This is suitable for a network where the number of nodes is comparatively less. Example of this is DSDV.

- REACTIVE PROTOCOL

Usually referred to as the on-demand routing protocol is the one in which the routes are determined on demand and then these are followed by the route maintenance process. Since the routes are maintained and updated on demand basis thus the routing tables are updated only whenever there is the need for the same, thereby avoiding the wastage of the bandwidth, however the latency in this case is more. Suitable for the network where the number of nodes is more. Example of this is AODV.

\section{- HYBRID PROTOCOL}

The involvement of both the proactive and reactive routing protocols in a single protocol is the hybrid routing protocol. In this protocol the set of nodes are aggregated in zones. Routing is initially established using the proactive approach and then they serve the demand for the additionally activated routes by following the reactive approach due to which it is suitable for larger networks. This protocol is required wherein multiple networks are there involving multiple number of nodes. Example is ZRP.

\section{METHODOLOGY USED}

As part of my research, I was given the design document of the routing protocol implemented for a high data rate L-band waveform developed for use by the Indian Navy. The design is based on the AODV protocol with suitable changes to cater for end user requirements and the applications being supported by the waveform. While the design of the routing protocol is proprietary and confidential, I was tasked with benchmarking the performance of the protocol against the well known and documented AODV protocol.

AODV stands for Ad-hoc on demand distance vector. A randomly generated route uses a route discovery process for its route determination in AODV routing protocol. This route discovery and maintenance that laid the basis for this protocol is supported by the help of three control packets viz. RREQ, RREP, RERR. The route discovery is done by using the both RREQ and RREP message cycle. When an originator node wants to send a message to the destination node for which it does not have any predetermined route then it will broadcast its RREQ control packet to its neighbouring nodes. The neighbours when received this RREQ packet will then check and update their routing tables in accordance with the originator node thereby setting a backward pointer towards the originating node in there routing tables. If the node receiving the RREQ is the desired destination node then it will send a RREP message back to the originator node otherwise it will again rebroadcast RREQ message to its neighbours. While setting a backward pointer toward the originating node the nodes also set a forward pointer to the destination node. A node selects a fresh enough route for route determination. A node having a sequence number greater than the previous one is considered for route discovery. In case the two nodes have the same sequence number than the node with lesser hop counts will be preferred more. In case of route failures a RERR message is sent to the source node and after receiving the RERR message the route discovery process is again initiated to compensate for the broken route and to find a new route for the same destination[4].

The methodology for the routing protocol of the SDR is similar. One major difference is the addressing scheme. The SDR waveform does not use IP addressing scheme because of the large overheads associated with inclusion of addresses in the headers of the control packets. The SDR waveform uses a proprietary addressing mechanism based on 1-bit MAC IDs assigned to all the nodes that are the part of the network. Also, the waveform caters for a maximum of 128 nodes. These nodes could be ships, submarines or aircraft. Due to the higher mobility of aircraft, these node types are given preferential treatment in terms of joining a network and relaying information. This is very relevant as aircraft not only move with much higher speeds, but also are available for only a short duration in the network. 


\section{SIMULATION RESULTS}

The performance of AODV routing protocol for SDR is simulated by using the MATLAB 2014a. The performance is recorded by taking different number of nodes. The MAC scheme used over here is CSMA/CA due to its capability to avoid the collisions in a network comprising of large number of nodes. The nodes are placed randomly in the network. The lists of some parameters are shown in Figl, that are varied in comparison to the AODV protocol to visualize the performance of AODV SDR for tactical communication. The following metrics are evaluated for SDR.

i. $\quad$ PDR (Packet Delivery Ratio)

It is the ratio of the packets received at the receiver to the packets that were sent by the transmitter. Better is the PDR better will be the performance of the protocol.

ii. $\quad$ Packet Loss

It is the packets lost during the transmission process due to congestion in the network.

iii. $\quad$ Routing Overhead

It is the total of control packets transmitted. More the overheads lower will be the efficiency of the adhoc network as the bandwidth consumption will be more for the transfer of data.

iv. Throughput

It is defined as the measure of the successful transmission rate, and is the ratio of the transmitted data packet to the simulation time. Better the throughput better is the transmission rate and better the performance is.

The results below benchmark the performance of AODV under standard conditions and AODV-SDR according to the requirements for SDR waveform. The simulation results are based on the following parameters.

Table 1: Parameter list for the simulations

\begin{tabular}{|c|c|c|c|}
\hline S.NO & PARAMETERS & AODV & AODV-SDR \\
\hline 1. & DATA RATE & $1 \mathrm{Mbps}$ & $6 \mathrm{Mbps}$ \\
\hline 2. & FRAME SIZE & $16 \mathrm{bits}$ & $10624 \mathrm{bits}$ \\
\hline 3. & TRANSMIT POWER & $-4 \mathrm{dBm}$ & $50 \mathrm{dBm}$ \\
\hline 4. & $\begin{array}{c}\text { RECEIVED SIGNAL } \\
\text { THRESHOLD }\end{array}$ & $-47 \mathrm{dBm}$ & $-35 \mathrm{dBm}$ \\
\hline 5. & $\begin{array}{c}\text { ACK TIMEOUT } \\
\text { RETRY LIMIT }\end{array}$ & $0.4 \mathrm{sec}$ & $5 \mathrm{sec}$ \\
\hline 7. & $\begin{array}{c}\text { SIMULATION } \\
\text { TIME(sec) }\end{array}$ & 50 & 1 \\
& \multicolumn{2}{|c|}{50} \\
\hline
\end{tabular}

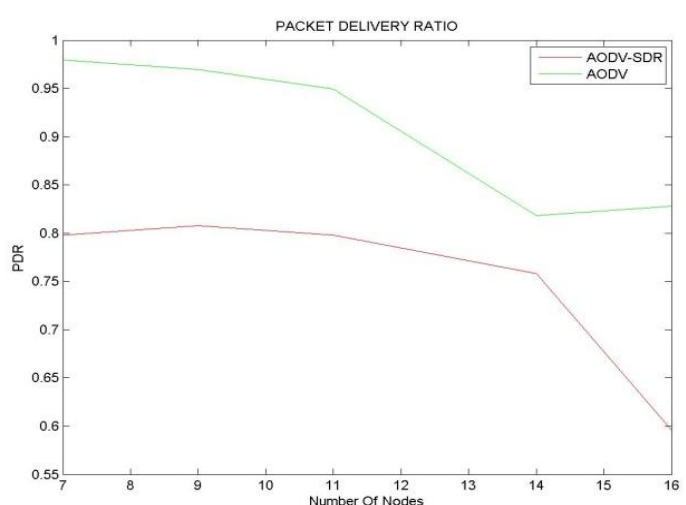

Fig1. PDR vs. Number of nodes

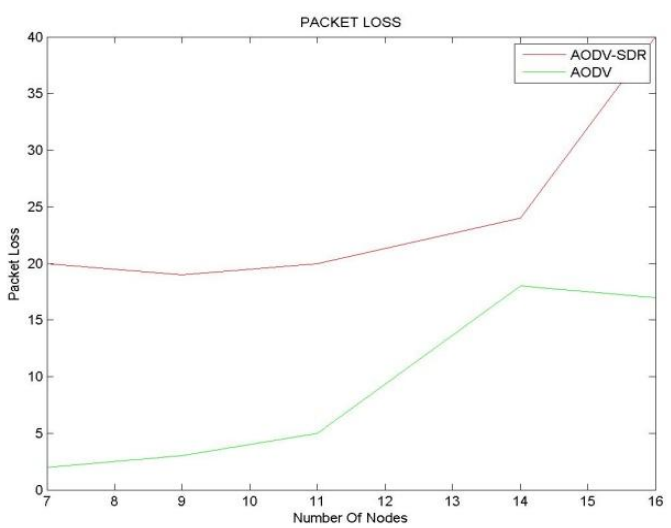

Fig2. Packet Loss vs. Number of nodes

Figure 2 and 3 shows the packet delivery ratio and packet loss with a network having more number of nodes. From the graph we can conclude that as the size of the network grows the number of received packets decreases leading to decrease in the PDR and increase in packet loss. This variation in the received packets has been observed due to the less number of retransmissions in AODV-SDR as compared to AODV.

Fig3. Routing overhead vs. Number of Nodes

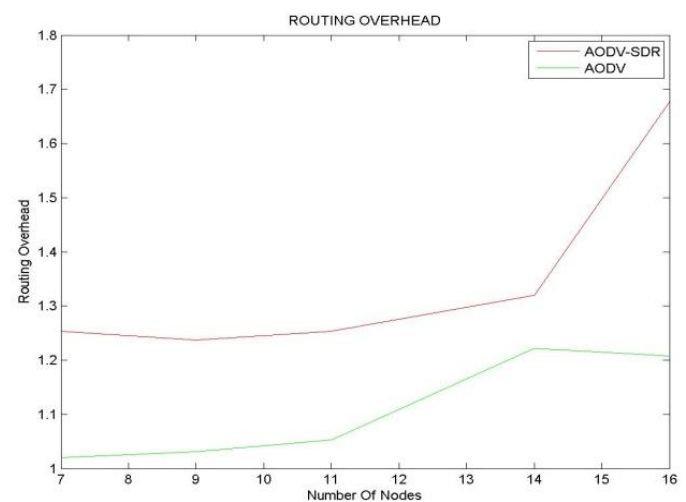

Figure 4 shows the overheads in a network which also increases with the network size. The increase in the overheads of AODV-SDR were found to be more than AODV because more number of control packets required to reach the destination as in packet loss is more in AODV-SDR. 


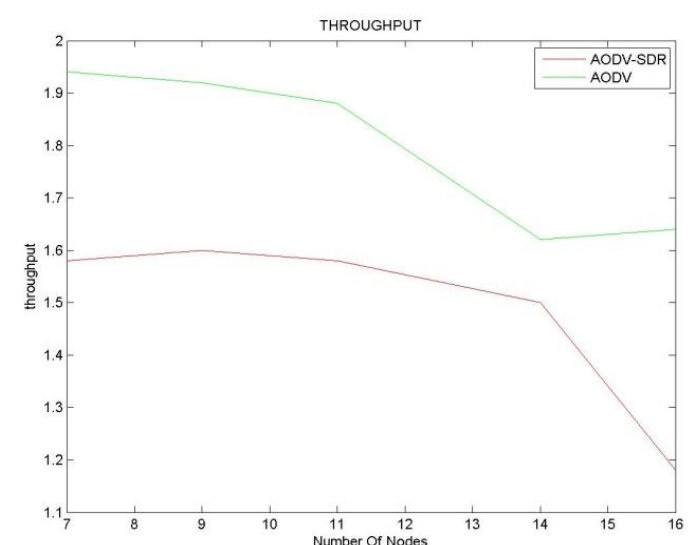

Fig4. Throughput vs. Number of Nodes

Figure 5 shows the variation of the throughput with nodes which degrades with the network size. The reason for this is because of the more routing information that will be required leading to the wastage of the bandwidth.

\section{CONCLUSION AND FUTURE WORK}

In this paper, the MANET Reactive protocol i.e AODV routing protocol has been implemented for SDR by using CSMA/CA protocol, with some modifications. From the above simulation results we have come across the conclusion that AODV when implemented for SDR does not give the better performance because of the loss of the packets. However, for better performance of the protocol with SDR the ACK timeout for the sender may decrease leading to the increase in the number of the received packets, thereby improving the performance up to an extent.

But if we talk about tactical communication the bandwidth and the routing overheads are the important parameters of consideration. From the graphs a clear observation can be made that as the network size grows the throughput for AODV-SDR decreases gradually whereas the routing overhead gradually increases. Thus we cannot use this technique for tactical communication wherein the number of nodes is more in a network.
For better performance in military communication hybrid (ZRP) [5] approach can be used; proactive [6] till a smaller size and reactive thereafter. As a further improvement, the design engineers have already implemented a dynamic TDMA as the MAC scheme. This not only obviates losses due to collisions but also ensures that the desired QoS for different applications riding on the SDR are met.

While the above mentioned protocol has already been implemented and is currently being tested prior to deployment, the same could not be shared due to nondisclosure and confidentiality clauses.

\section{ACKNOWLEDGMENTS}

For the successful completion of this work, I want to thank my guides, Ms. Amanpreet kaur, Assistant professor at ITM University, Gurgaon, Haryana and Mr. Dhiraj Khanna from Ministry of Defence. Without their able guidance this work would not have been possible in this manner.

\section{REFERENCES}

[1] Mark W. Chamberlain, "A SOFTWAREDEFINED BY RADIO", Harris Corporation, RF Communications Division Rochester, New York

[2] Priyanka Goyal, Vinti Parmar, Rahul Rishi, "MANET: Vulnerabilities, Challenges, Attacks, Application", Research Scholar, Dept. of CSE, Technological Institute of Textile and Science, Bhiwani, Haryana, India.

[3] MadhaviW.Subbarao, "Ad Hoc Networking Critical Features and Performance Metrics", Wireless Communications Technology Group, NIST October 7, 1999.

[4] Clifton Lin, "AODV Routing Implementation for Scalable Wireless Ad-Hoc Network Simulation (SWANS)".

[5] Zygmunt J. Haas, Senior Member, IEEE and Marc R. Pearlman, Member, IEEE, "The Performance of Query Control Schemes for the Zone Routing Protocol".

[6] Guoyou He, "Destination-Sequenced Distance Vector (DSDV) Protocol", Networking Laboratory Helsinki University of Technology. 